\title{
Depression mediates cutaneous body image and facial appearance dissatisfaction in insomnia
}

\author{
Umair Akram ${ }^{1,2}$ [ Kamila Irvine ${ }^{3}$
}

Received: 11 September 2019 / Accepted: 11 January 2020 / Published online: 23 January 2020

(c) The Author(s) 2020

\begin{abstract}
This study examined the relationship between dissatisfaction with cutaneous body image and facial appearance with symptoms of insomnia whilst incorporating the mediating role of anxiety and/or depression after accounting for co-morbid sleep disorder symptoms. Participants $(n=241)$ completed online measures assessing insomnia symptoms, anxiety and depression symptoms, and satisfaction with cutaneous body image and facial appearance. Symptoms of insomnia were independently related to greater dissatisfaction with cutaneous body image and facial appearance in univariate analyses. However, linear regression analyses determined these relationships to be partially mediated by depression, but not anxiety. Expanding on prior research, these findings suggest that whilst increased symptoms of insomnia may influence dissatisfaction with cutaneous and facial features, these relationships may be partially attributed to the experience of depressive symptoms often co-morbid with both insomnia and dermatological complaints. Treatment approaches for individuals with insomnia may benefit from targeting and improving negatively appraised aspects of physical self-perception.
\end{abstract}

Keywords Insomnia $\cdot$ Anxiety $\cdot$ Depression $\cdot$ Cutaneous body image $\cdot$ Face perception

\section{Introduction}

Symptoms of insomnia, including difficulty initiating and/ or maintaining sleep, or early morning awakenings with an inability to return to sleep, and impaired daytime functioning, are highly prevalent affecting $30 \%$ of the general population [1-3]. It has been evidenced that individuals experiencing insomnia frequently present difficulty with emotion regulation and self-perception [4-6] and display self-focused attention on awakening and throughout the day to assess and confirm the perceived poor nature of their sleep [7]. According to prominent cognitive models of the disorder, this pattern of thinking may perpetuate and maintain

Umair Akram

u.akram@shu.ac.uk

1 Department of Psychology, Sociology and Politics, Sheffield Hallam University, Collegiate Crescent, Sheffield, South Yorkshire S10 2BP, UK

2 Nuffield Department of Clinical Neurosciences, University of Oxford, Oxford, UK

3 Department of Psychology, University of Lincoln, Lincoln, UK insomnia by potentiating cognitive arousal, distress, and negative thoughts concerning sleep [8].

It has been previously demonstrated that individuals with insomnia and poor sleepers negatively appraise aspects of their physical appearance $[9,10]$. Specifically, Gupta et al. [9] note that dissatisfaction with one's own cutaneous body image, the mental perception of the appearance of the skin, hair and nails, appears to be directly related to insomnia symptoms, whereas Oyetakin-White et al. [10] demonstrated that poor sleepers show greater self-perceived dissatisfaction with aspects of their facial appearance (e.g. complexion, skin age) when compared with normal sleepers. This evidence suggests that poor sleep and symptoms of insomnia confound an individual's appraisal of physical self-perception. To that end, improving physical self-perception may attenuate selective attention for cues consistent with poor sleep, which perpetuate negative-thought cycles highlighted in cognitive models of insomnia [8]. However, considering the early stage of research in this area, little is known about underlying and potentially treatable mechanisms which negatively influence cutaneous body image perception in insomnia.

Major depressive disorder appears to be the most common psychiatric comorbidity in relation to dermatological 
complaints [11]. It has been proposed that feelings of worthlessness may at least partially manifest as low self-esteem, body image dissatisfaction with a particular focus on the face, and delusions of disfigurement amongst those who also present with psychotic features [11]. Similarly, socially anxious individuals appear to be overly self-conscious with regard to excessive blushing/flushing and hyperhidrosis [12]. With that in mind, it is well established that symptoms of anxiety and depression commonly co-occur with insomnia [13] and recently have been evidenced to mediate the relationship between insomnia and self-disgust [6]. Therefore, it is possible that the psychological factors mediating the relationship between dissatisfaction with cutaneous body image and facial appearance and insomnia symptoms may include anxiety and depression.

The present study aimed to investigate the potential mediating role of anxiety and/or depression on the relationship between insomnia symptoms and dissatisfaction with cutaneous body image and facial appearance after accounting for other co-morbid sleep disorders. Specifically, we aimed to determine whether symptoms of insomnia would be related to greater dissatisfaction with cutaneous body image, facial appearance and skin-age, and whether any confirmed relationships are mediated by symptoms of anxiety and/or depression.

\section{Materials and methods}

Ethical approval was granted by the Northumbria University Research Ethics Committee. This experiment was conducted in accordance with the Declaration of Helsinki, and all participants gave their informed consent before participation.

\section{Sample and procedure}

A cross-sectional online questionnaire-based study was implemented, comprising of questions designed to measure symptoms of insomnia, anxiety and depression in addition to satisfaction with cutaneous body image, facial appearance and skin age. The survey was advertised to members of the general population through social media, "call for participants' (website), and students at Northumbria University. Initially, 280 participants responded to the online survey, and 241 respondents provided complete data. Participants who indicated that they conducted shift work, suffered from a disorder of the central nervous system, or had a prior head injury or reported symptoms of a sleep disorder other than insomnia were excluded from analysis $(n=34)$. This resulted in a final sample of 207 participants (mean age $=24.67 \pm 7.73$, age range $18-56,80 \%$ female) who were entered into the analysis.

\section{Measures}

\section{Sleep condition indicator}

Insomnia symptoms were assessed using sleep condition indicator (SCI), a clinical screening tool developed to appraise insomnia symptoms against the DSM- 5 criteria for Insomnia Disorder [14]. The scale consists of eight items each scored between 0 and 4 designed to examine insomnia symptomology during the last month. Specifically, questions pertain to sleep onset latency, awakenings during the night, perceived sleep quality, impairment to daytime functioning and symptom persistence. Items are summed to create a total score between 0 and 32, with lower scores indicating greater insomnia symptom severity. Current assessment of internal consistency yielded a Cronbach's alpha of 0.91 .

\section{Anxiety and depression}

Symptoms of anxiety and depression were assessed using the Hospital Anxiety and Depression Scale (HADS: [15]), consisting of 14 items (seven for each anxiety and depression) scored between 0 and 3, with a maximum score of 21 for each subscale. Higher scores on each subscale represent greater anxiety (HADS-A) and depression (HADSD). Both subscales demonstrated good internal consistency (Cronbach's $\alpha$ of 0.89 and 0.78 for anxiety and depression respectively).

\section{Cutaneous body image}

Cutaneous body image was assessed using the Cutaneous Body Image Scale [16]. The CBI comprises seven items on a 10-point Likert scale that examine satisfaction with skin appearance, complexion, facial appearance, hair and nails. Ratings of: 0 indicate 'not at all'; $1-3$ indicate 'mildly'; 4-6 indicate moderately; and 7-9 indicate 'a great deal'. The mean of the seven ratings indicates the final score. Higher scores represent greater satisfaction; lower scores represent greater dissatisfaction. Current assessment of internal consistency yielded a Cronbach's alpha of 0.91 .

\section{Facial appearance}

Satisfaction with facial appearance (FA) was examined using the measure developed by Oyetakin-White et al. [10]. Six items are used to examine individuals' feelings about their facial appearance (e.g. 'how healthy do you think your face looks', 'overall, how satisfied are you with your complexion'). Items are rated on a scale from 1 (poor) to 5 (excellent). The summation of each item indicated the final total 
score, with higher scores representing greater satisfaction. Current assessment of internal consistency yielded a Cronbach's alpha of 0.86 .

\section{Statistical analyses}

Pearson's bivariate correlations examined associations between insomnia symptoms, anxiety, depression, and satisfaction with cutaneous body image and facial appearance. This was followed by a series of hierarchical linear regression analyses (using the enter method), with cutaneous body image and facial appearance satisfaction as dependent variables, to determine whether any significant associations were mediated by anxiety and/or depression after controlling for age and sex. All statistical analyses were performed using Jamovi version 1.02 (The Jamovi Project, 2019). Significance was considered at the $p<0.05$ level (two-tailed).

\section{Results}

Mean scores for the final sample are presented in Table 1. SCI scores were positively related to cutaneous body image $(r=0.48, p=0.001)$ and facial appearance $(r=0.42$, $p=0.001)$ satisfaction, and negatively related to anxiety $(r=-0.40, p=0.001)$ and depression $(r=-0.52, p=0.001)$. Moreover, anxiety and depression were negatively related to satisfaction with cutaneous body image (anxiety: $r=-0.31, p=0.001$; depression: $r=-0.41, p=0.001)$ and facial appearance (anxiety: $r=-0.28, p=0.001$; depression: $r=-0.40, p=0.001$ ). Finally, cutaneous body image and facial appearance satisfaction were positively related $(r=0.81, p=0.001)$.

Linear regression analyses indicated that dissatisfaction with cutaneous body image and sex (step 2: 24\% total variance explained) significantly predicted insomnia symptoms after controlling for age and sex (step 1: $0.05 \%$ variance). After accounting for anxiety and depression, insomnia and depressive symptoms remained significant predictors in the subsequent steps (step 3: $25 \%$. variance step 4: $28 \%$. variance; see Table 2a). Similarly, dissatisfaction with facial appearance and sex (step 2: $18 \%$ total variance explained) significantly predicted insomnia symptoms after controlling for age and sex (step 1: $0.02 \%$ variance). After accounting for anxiety and depression, insomnia and depressive symptoms remained significant predictors in the subsequent steps (step 3: $25 \%$. variance step 4: $28 \%$. variance; see Table 2b).

The mediating effects of depression were further examined using the MEDMOD plugin for Jamovi. Bootstrapping with 1000 bias-corrected and accelerate resamples and 95\% confidence intervals were used, and the Sobel test $(z)$ was used to indicate the hypothesized mediation effects. As demonstrated in Table 3, the results demonstrated significant direct effects between insomnia symptoms with cutaneous body image and facial appearance satisfaction. Moreover, indirect effects of insomnia symptoms on cutaneous body image and facial appearance (respectively) via depression were also evidenced. Therefore, whilst cutaneous body image and facial appearance dissatisfaction were related to increased insomnia symptoms, these relationships appear to be partially mediated by symptoms of depression.

\section{Discussion}

The present study examined the mediating role of anxiety and depression on the relationship between insomnia symptoms and dissatisfaction with cutaneous body image and facial appearance. The current results provide further evidence that dissatisfaction with cutaneous body image and facial appearance are related to symptoms of insomnia. In addition, our findings demonstrate that these relationships appear to be partially mediated by depression but not anxiety.

Individuals with insomnia are generally less healthy compared to those who sleep normally [17], and this may be reflected in the appearance of their skin. Indeed, various mechanisms can promote physiological changes which influence an individual's self-perception. Tiredness, a commonly reported feature of insomnia [18] for example, can
Table 1 Mean scores (and standard deviations) and sex differences for each measure

\begin{tabular}{lrrrrl}
\hline & $\begin{array}{l}\text { Whole sample } \\
\text { Mean } \pm \mathrm{SD}\end{array}$ & \multicolumn{1}{l}{$\begin{array}{l}\text { Female } \\
\text { Mean } \pm \mathrm{SD}\end{array}$} & \multicolumn{1}{l}{$\begin{array}{l}\text { Male } \\
\text { Mean } \pm \mathrm{SD}\end{array}$} & & $\mathrm{p}$ \\
\hline Anxiety & $7.82 \pm 4.36$ & $8.51 \pm 4.76$ & $5.13 \pm 3.41$ & -4.32 & $0.001^{* *}$ \\
Depression & $4.07 \pm 3.30$ & $4.28 \pm 3.35$ & $3.22 \pm 2.85$ & -1.86 & 0.064 \\
Insomnia symptoms & $20.74 \pm 8.17$ & $19.82 \pm 7.94$ & $24.17 \pm 8.22$ & 3.14 & $0.002^{*}$ \\
Cutaneous body image satisfaction & $4.84 \pm 2.07$ & $4.64 \pm 2.01$ & $5.74 \pm 2.04$ & 3.13 & $0.002^{*}$ \\
Facial appearance satisfaction & $19.07 \pm 4.90$ & $18.82 \pm 4.71$ & $20.26 \pm 5.41$ & 1.71 & 0.089 \\
\hline
\end{tabular}

Anxiety and depression, hospital anxiety and depression scale; insomnia symptoms; sleep condition indicator

Note: $*$ Sig at $<0.01 * *<0.001$ 
Table 2 Linear regression analyses with cutaneous body image (a) and facial appearance satisfaction (b) as dependent variables; age, sex, insomnia symptoms, anxiety and depression as predictors

\begin{tabular}{|c|c|c|c|c|}
\hline Predictors & $R 2$ & $\beta$ & $t$ & Sig. \\
\hline \multicolumn{5}{|l|}{ (a) } \\
\hline Step 1 & 0.06 & & & \\
\hline Age & & 0.01 & 0.17 & 0.87 \\
\hline Sex & & -0.25 & -3.62 & $0.001 * *$ \\
\hline Step 2 & 0.26 & & & \\
\hline Age & & 0.09 & 1.50 & 0.14 \\
\hline Sex & & -0.14 & -2.23 & $0.03 *$ \\
\hline Insomnia symptoms & & 0.46 & 7.20 & $0.001 * *$ \\
\hline Step 3 & 0.27 & & & \\
\hline Age & & 0.09 & 1.45 & 0.15 \\
\hline Sex & & -0.11 & 6.06 & 0.09 \\
\hline Insomnia symptoms & & 0.41 & -1.95 & $0.001 * *$ \\
\hline Anxiety & & -0.13 & 4.61 & 0.05 \\
\hline Step 4 & 0.30 & & & \\
\hline Age & & 0.11 & 1.80 & 0.07 \\
\hline Sex & & -0.12 & -1.95 & 0.05 \\
\hline Insomnia symptoms & & 0.33 & 4.62 & $0.001 * *$ \\
\hline Anxiety & & -0.05 & -0.65 & 0.52 \\
\hline Depression & & -2.20 & -2.86 & $0.005^{*}$ \\
\hline \multicolumn{5}{|l|}{ (B) } \\
\hline Step 1 & 0.02 & & & \\
\hline Age & & -0.20 & -0.29 & 0.78 \\
\hline Sex & & -0.15 & -2.21 & $0.03 *$ \\
\hline Step 2 & 0.18 & & & \\
\hline Age & & 0.05 & 0.87 & 0.42 \\
\hline Sex & & -0.05 & -0.87 & 0.38 \\
\hline Insomnia symptoms & & 0.41 & 6.16 & $0.001 * *$ \\
\hline Step 3 & 0.20 & & & \\
\hline Age & & 0.05 & 0.76 & 0.45 \\
\hline Sex & & -0.02 & -0.34 & 0.73 \\
\hline Insomnia symptoms & & 0.36 & 5.01 & $0.001 *$ \\
\hline Anxiety & & -0.16 & -2.19 & $0.03 *$ \\
\hline Step 4 & 0.24 & & & \\
\hline Age & & 0.07 & 1.14 & 0.26 \\
\hline Sex & & -0.04 & -0.57 & 0.57 \\
\hline Insomnia symptoms & & 0.27 & 3.53 & $0.001 * *$ \\
\hline Anxiety & & -0.06 & -0.76 & 0.45 \\
\hline Depression & & -0.25 & -3.17 & $0.002 *$ \\
\hline
\end{tabular}

Note: $*$ Sig at $<0.05, * *<0.01$
Table 3 Examination of the mediating effect of depression, with cutaneous body image and facial appearance satisfaction as dependent variables (IV) and depression as a mediator (DV) lead to relaxing of the muscles intrinsically altering facial expression [19]. Moreover, blood flow to the skin is promoted during sleep, and evidence suggests skin blood coloration occurs with sleep loss such that the face eventually appears paler [20]. With that in mind, photographs of sleep deprived individuals are rated as less attractive, less healthy, more tired, and unsociable relative to when well rested [21, 22]. More specifically, sleep-deprived individuals appear to present specific dermatological characteristics relating to tiredness (e.g. wrinkles/fine lines and bags around the eyes, dropped corners of the mouth, and heavy eyes [22]). Therefore, it may be theorised that individuals presenting insomnia symptoms are more sensitive to physiological and dermatological changes which influence their self-perception of cutaneous body image and facial appearance in a negative manner, leading to dissatisfaction with own appearance.

By definition, insomnia involves subjective reports of sleep disturbance [1]. However, objective examination of sleep in this population often fails to provide evidence to support the extent of reported symptomology [23-26]. Whilst studies examining the physiological and dermatological changes occurring following extreme sleep deprivation, [21, 22] people with insomnia may not present alternations to the same degree. Rather, falling in between a state of sleep sated and sleep deprived [27]. Considering this, we may theorise that mechanisms underlying insomnia (e.g. cognitive biases) serve to accentuate the negative nature of judgments pertaining to physical self-perception $[8,27]$. Indeed, people with insomnia often selectively attend to and monitor internal and external bodily cues/ sensations upon waking, as well as throughout the day, for signs consistent with a poor night of sleep (e.g. heavy eyes, poor complexion $[7,28])$. Qualitative examination evidences that upon awakening, those with insomnia frequently report internal sensations of feeling tired and unrefreshed, with aspects of body perceived as sore and heavy [28], whereas externally, selective attention was predominately oriented towards facial appearance, with an emphasis on the eyes and surrounding region. Here, facial dissatisfaction was highly prevalent, with eyes described as being heavy and sunken, red or yellow in colour, and accompanied with large bags underneath. However, the authors note that occurrence of this throughout the course of the day was largely contingent on convenience and

\begin{tabular}{llllllr}
\hline IV & Mediator & DV & $\begin{array}{l}\text { Total effect (c } \\
\text { path) }\end{array}$ & $\begin{array}{l}\text { Direct effect } \\
\left(c^{\prime} \text { path) }\right.\end{array}$ & \multicolumn{2}{l}{ Total indirect effect } \\
\cline { 5 - 6 } & & & Point est. & $95 \%$ CI \\
\hline Insomnia & Depression & CBI & $7.54 * *$ & $4.23 * *$ & $0.030^{*}$ & $0.008,0.051$ \\
Insomnia & Depression & FA & $5.42 * *$ & $2.84 *$ & $0.082 *$ & $0.030,0.142$ \\
\hline
\end{tabular}

Mediation model, 1000 bootstrap samples

Note: $*$ Sig at $<0.01, * *<0.001$ 
opportunity (e.g. using the mirror in bathroom breaks) [28]. Moreover, experimental evidence objectively demonstrates heightened attention towards and misinterpretation of dermatologic cues pertaining to tiredness in this population $[4,29,30]$. As such, biases of attention towards cutaneous and facial features amongst those experiencing insomnia symptoms are likely to influence the negative orientation of their interpretation, confirming for at least the individual, the presence of a sleep disturbance.

Whilst symptoms of insomnia influence dissatisfaction with cutaneous and facial features, the current outcomes highlight the mediating role of depressive symptoms often co-morbid with insomnia [31] and dermatological complaints [16]. The inability to cope with daytime consequences of insomnia (e.g. irritability, interpersonal difficulties) is considered to trigger the onset of depression (Staner, 2010). In turn, accentuation of pre-existing adverse cognitive-emotional processes (i.e. worry, rumination, emotion dysregulation) and negatively valanced self-conscious emotions (e.g. self-disgust) may increase and further internalise body image dissatisfaction [32]. Ultimately, the culmination symptom cycle to fuel behavioural alterations which maintain insomnia. For example, avoiding social commitments due, not only, to perceived daytime impairments (e.g."I should stay in and go to bed earlier to catch up with sleep, otherwise I would not focus at work tomorrow"), but also negative self-appraisal (e.g."I look too tired to go out") and depressive symptoms (e.g."I don't feel like socialising"). Working to improve an individual's self-perception should be considered a focal point of treatment. Initiation of a more accurate self-perception may consequently eliminate a maintaining factor of insomnia (i.e. the propensity to negatively interpret physical cues which reflect poor sleep). To that end, feedback relating to a self-misperception of facial tiredness in insomnia has tentatively functioned to improve selfperception such that individuals later perceived themselves as appearing less tired [4]. Moving forward, research should further explore possible interventions aiming to normalise additional characteristics of facial appearance and cutaneous body image in insomnia. Additionally, the role of selfconscious emotions related to insomnia and depression in predicting body image dissatisfaction should be examined.

Interestingly, the relationship between insomnia symptoms and dissatisfaction with cutaneous body image and facial appearance was not mediated by symptoms of anxiety. Whilst depression commonly co-occurs alongside dermatological complaints $[11,33]$, anxiety in this context remains often limited to dermatological cues $[12,33]$. The broad measure of anxiety used in the present study does not examine such cues and, therefore, may explain why anxiety failed to mediate the relationship between insomnia symptoms and dissatisfaction with cutaneous body image and facial appearance.
Although the co-morbid sleep disorders were accounted for, a number of caveats should be highlighted in relation to the current study. First, the current sample consisted largely of female participants and, therefore, the present findings may not be fully generalizable to males. That said, women are more likely than men to experience symptoms of insomnia [34]. Moreover, examination of sex differences found greater CBI (but not facial appearance) dissatisfaction amongst females. However, regression analysis failed to evidence an individual's sex as a significant predictor of CBI and FA dissatisfaction, respectively. Next, the current outcomes cannot be extrapolated to individuals meeting diagnostic criteria for insomnia. However, the use of a nonclinical sample can be viewed as a logical first step towards identifying the mediating role of depression. Finally, the cross-sectional nature of the study limits inferences relating to causality and directionality. Moving forward, the current research questions should be further examined longitudinally amongst a larger and more balanced sample.

In sum, we evidence dissatisfaction with cutaneous body image and facial appearance to be related to insomnia symptoms. However, these relationships appear to be partially mediated by depression. Whilst cognitive behavioural therapy for insomnia remains the first-line treatment approach for insomnia, also holding therapeutic effects for those experiencing co-morbid depression [35], those who are treatment-resistant may benefit from targeting and improving negatively appraised aspects of physical self-perception.

Author contributions The experiment was designed and conceived by UA. Data were collected and analysed by UA. The manuscript was written by UA and KI.

Funding No funding was received for this research

\section{Compliance with ethical standards}

Conflict of interest All authors declare that there are no conflicts of interest.

Ethical standards Ethical approval was granted by the the Northumbria University Research Ethics Committee. This experiment was conducted in accordance with the Declaration of Helsinki, and all participants gave informed consent before participation.

Open Access This article is licensed under a Creative Commons Attribution 4.0 International License, which permits use, sharing, adaptation, distribution and reproduction in any medium or format, as long as you give appropriate credit to the original author(s) and the source, provide a link to the Creative Commons licence, and indicate if changes were made. The images or other third party material in this article are included in the article's Creative Commons licence, unless indicated otherwise in a credit line to the material. If material is not included in the article's Creative Commons licence and your intended use is not permitted by statutory regulation or exceeds the permitted use, you will 
need to obtain permission directly from the copyright holder. To view a copy of this licence, visit http://creativecommons.org/licenses/by/4.0/.

\section{References}

1. American Psychiatric Association. Diagnostic and statistical manual of mental disorders (DSM-5 ${ }^{\circledR}$ ). American Psychiatric Pub; 2013.

2. Espie CA, Kyle SD, Hames P, Cyhlarova E, Benzeval M. The daytime impact of DSM-5 insomnia disorder: comparative analysis of insomnia subtypes from the Great British Sleep Survey. J Clin Psychiatry. 2012;73(12):e1478-84.

3. Morin CM, LeBlanc M, Daley M, Gregoire JP, Merette C. Epidemiology of insomnia: prevalence, self-help treatments, consultations, and determinants of help-seeking behaviors. Sleep Med. 2006;7(2):123-30.

4. Akram U, Ellis JG, Myachykov A, Barclay NL. Misperception of tiredness in young adults with insomnia. J Sleep Res. 2016;25(4):466-74.

5. Baglioni C, Spiegelhalder K, Lombardo C, Riemann D. Sleep and emotions: a focus on insomnia. Sleep Med Rev. 2010;14(4):227-38.

6. Ypsilanti A, Lazuras L, Robson A, Akram U. Anxiety and depression mediate the relationship between self-disgust and insomnia disorder. Sleep Health. 2018;4(4):349-51.

7. Semler CN, Harvey AG. Misperception of sleep can adversely affect daytime functioning in insomnia. Behav Res Ther. 2005;43(7):843-56.

8. Harvey AG. A cognitive model of insomnia. Behav Res Ther. 2002;40(8):869-93.

9. Gupta MA, Gupta AK, Knapp K. Dissatisfaction with cutaneous body image is directly correlated with insomnia severity: a prospective study in a non-clinical sample. J Dermatol Treat. 2015;26(2):193-7.

10. Oyetakin-White P, Suggs A, Koo B, Matsui MS, Yarosh D, Cooper KD, Baron ED. Does poor sleep quality affect skin ageing? Clin Exp Dermatol. 2015;40(1):17-22.

11. Levenson JL. The American Psychiatric Publishing Textbook of Psychosomatic Medicine: psychiatric care of the medically ill. American Psychiatric Pub; 2011.

12. Gupta MA, Gupta AK. The use of antidepressant drugs in dermatology. J Eur Acad Dermatol Venereol. 2001;15(6):512-8.

13. Ford DE, Kamerow DB. Epidemiologic study of sleep disturbances and psychiatric disorders: an opportunity for prevention? JAMA. 1989;262(11):1479-84.

14. Espie CA, Kyle SD, Hames P, Gardani M, Fleming L, Cape J. The sleep condition indicator: a clinical screening tool to evaluate insomnia disorder. BMJ Open. 2014;4(3):e004183.

15. Zigmond AS, Snaith RP. The hospital anxiety and depression scale. Acta Psychiatr Scand. 1983;67(6):361-70.

16. Gupta MA, Gupta AK. Evaluation of cutaneous body image dissatisfaction in the dermatology patient. Clin Dermatol. 2013;31(1):72-9.

17. Taylor DJ, Lichstein KL, Durrence HH. Insomnia as a health risk factor. Behav Sleep Med. 2003;1(4):227-47.
18. Balter MB, Uhlenhuth EH. The beneficial and adverse effects of hypnotics. J Clin Psychiatry. 1991;52:16-23.

19. Enoka RM, Stuart DG. Neurobiology of muscle fatigue. J Appl Physiol. 1992;72(5):1631-48.

20. Kräuchi K, Wirz-Justice A. Circadian clues to sleep onset mechanisms. Neuropsychopharmacology. 2001;25(S1):S92.

21. Axelsson J, Sundelin T, Ingre M, Van Someren EJ, Olsson A, Lekander M. Beauty sleep: experimental study on the perceived health and attractiveness of sleep deprived people. BMJ. 2010;341:c6614.

22. Sundelin T, Lekander M, Kecklund G, Van Someren EJ, Olsson A, Axelsson J. Cues of fatigue: effects of sleep deprivation on facial appearance. Sleep. 2013;36(9):1355-60.

23. Perlis ML, Merica H, Smith MT, Giles DE. Beta EEG activity and insomnia. Sleep Med Rev. 2001;5(5):365-76.

24. Tang NK, Harvey AG. Altering misperception of sleep in insomnia: behavioral experiment versus verbal feedback. J Consult Clin Psychol. 2006;74(4):767.

25. Tang NK, Harvey AG. Correcting distorted perception of sleep in insomnia: a novel behavioural experiment? Behav Res Ther. 2004;42(1):27-39.

26. Van Den Berg JF, Van Rooij FJ, Vos H, Tulen JH, Hofman A, Miedema HM, Neven AK, Tiemeier H. Disagreement between subjective and actigraphic measures of sleep duration in a population-based study of elderly persons. J Sleep Res. 2008;17(3):295-302.

27. Akram U. The face of tiredness in insomnia from the self-perspective: a focus on attentional and interpretative biases. J Sleep Res. 2018;27(3):e12657.

28. Akram U, Kay S, Fonquerine Z. Qualitative examination of daytime monitoring and selective attention in insomnia. Sleep Vigil. 2018;2(2):149-55.

29. Akram U, Ellis JG, Myachykov A, Barclay NL. Preferential attention towards the eye-region amongst individuals with insomnia. J Sleep Res. 2017;26(1):84-91.

30. Akram U, Robson A, Ypsilanti A. Sleep-related attentional bias for faces depicting tiredness in insomnia: evidence from an eyetracking study. J Clin Sleep Med. 2018;14(06):959-65.

31. Staner L. Comorbidity of insomnia and depression. Sleep Med Rev. 2010;14(1):35-46.

32. Ypsilanti A, Lazuras L, Powell P, Overton P. Self-disgust as a potential mechanism explaining the association between loneliness and depression. J Affect Disord. 2019;243:108-15.

33. Fried RG, Gupta MA, Gupta AK. Depression and skin disease. Dermatol Clin. 2005;23(4):657-64.

34. Zhang B, Wing YK. Sex differences in insomnia: a meta-analysis. Sleep. 2006;29(1):85-93.

35. Taylor DJ, Pruiksma KE. Cognitive and behavioural therapy for insomnia (CBT-I) in psychiatric populations: a systematic review. Int Rev Psychiatry. 2014;26(2):205-13.

Publisher's Note Springer Nature remains neutral with regard to jurisdictional claims in published maps and institutional affiliations. 